\title{
Constraints on Mixing Processes from Abundance Anomalies
}

\author{
G. Michaud, J. Richer, and O. Richard \\ Département de physique, Université de Montréal, Montréal, $P Q$, \\ Canada, H3C 3J7; CEntre de Recherche en Calcul Appliqué (CERCA), \\ 5160 boul. Décarie, bureau 400, Montréal, PQ, Canada, H3X $2 H 9$
}

\begin{abstract}
Abundance anomalies in the Sun, main sequence F, A and B stars, turnoff Pop II stars, the horizontal branch and white dwarfs are caused at least partly by particle transport processes. Detailed evolutionary models have been calculated for most of those objects taking into account the gravitational settling, thermal diffusion and radiative accelerations of 28 isotopes (24 atomic species). These will be used together with the observed abundances to put constraints on the mixing that rotationnally induced turbulence may lead to. The link between abundance anomalies and rotation on the HB is explained by the variation with $\log g$ of the ratio of the Eddington-Sweet and atomic diffusion velocities.
\end{abstract}

\section{Atomic Diffusion in Stellar Evolution}

The effects of rotation on particle transport in stars are not yet fully determined from first principles. We take the approach to determine the abundance anomalies that should be expected if there were no rotation. The series of models without rotation is then used as a reference series of models to evaluate the effects of rotation on internal stellar transport.

Atomic diffusion is a basic physical transport process that always occurs and is important whenever macroscopic motions, if present, are not rapid enough to wipe out its effects. The only series of models calculated up to now that include particle transport in a self-consistent manner are those starting with Turcotte et al. (1998b) where all effects of atomic diffusion are taken into account. They were made possible by the recent availability of large atomic data bases that include the atomic data needed to calculate radiative accelerations throughout stellar models. Using this data the radiative accelerations and Rosseland opacities are continuously calculated during evolution as the relative concentrations change (Richer et al. 1998). They are included in the particle transport equations leading to 56 ( 28 chemical species and 2 equations per species) non-linear coupled differential equations (Burgers, 1969; Turcotte et al. 1998b). For Pop I stars, models were evolved from the zero age main sequence to the bottom (or middle in some cases) of the giant branch for stars with mass from 0.5 to $4.0 M_{\odot}$. For Pop II stars of 0.5 to $1.2 M_{\odot}$, models were calculated for $Z=0.017 \times 10^{-4}$ to $0.0068([\mathrm{Fe} / \mathrm{H}]=-4.31$ to -0.71$)$, from the pre-main sequence to the lower part of the giant branch. 
In this review, we briefly describe the anomalies and structural changes to be expected in models calculated from first principles in both Pop I and Pop II stars. We then evaluate the turbulence, possibly caused by rotation, that would be required to reduce the anomalies to the observed values. For those cases where turbulence is included, the particle transport equations were described in Richer et al. (2000) and Richard et al. (2001). Our aim is to investigate the size of turbulent transport coefficients that observed abundance anomalies require if turbulence is the main process perturbing our evolutionary models obtained from first principles.

\section{Population I}

In $1 M_{\odot}$ stars, helioseismology has confirmed the gravitational settling of $\mathrm{He}$ in the Sun's external regions (Guzik \& Cox 1992; Christensen-Dalsgaard et al. 1993; Bahcall et al. 1995; Guenther et al. 1996; Richard et al. 1996; Brun et al. 1999). Proffitt \& Michaud (1991) introduced a turbulent transport coefficient to compete with He settling and at the same time cause enough Li destruction to explain the observed surface solar $\mathrm{Li}$ abundance. The turbulent transport that reduces sufficiently the $\mathrm{Li}$ abundance simultaneously reduces $\mathrm{He}$ settling by $1 / 3$ and the Be surface abundance by about a factor of 2 . It is compared in Section 4 . to the turbulent transport coefficient in Pop II stars. Basu (1997) has shown that weak turbulence below the solar convection zone also improves agreement with the solar pulsation spectrum. Other parametrizations of turbulence were introduced for the Sun by Richard et al. (1996) and Brun et al. (1999).

Even in $1 M_{\odot}$ models, Turcotte et al. (1998b) showed that $g_{R}$ were nearly equal to $g$ for many metals below the convection zone so modifying the surface abundances. However in stars of $1.3 M_{\odot}$ or more, the overabundances caused by $g_{R}$ become widespread. In solar metallicity stars with $T_{\text {eff }}>7000 \mathrm{~K}$, selective radiative acceleration causes an accumulation of iron-peak elements, and especially $\mathrm{Fe}$, at a temperature of about $200000 \mathrm{~K}$ where these elements are a major contributor to opacity (Turcotte et al., 1998a; Richard et al., 2001). Those F and A stars that rotate rapidly $\left(\geq 100 \mathrm{~km} \mathrm{~s}^{-1}\right)$ do not show abundance anomalies that atomic diffusion leads to. However anomalies appear to be present in the $\mathrm{B}, \mathrm{A}$ and $\mathrm{F}$ stars that rotate more slowly than $100 \mathrm{~km} \mathrm{~s}^{-1}$ (Abt, 2000) leading to the HgMn and AmFm stars.

\subsection{AmFm Stars}

Richer et al. (2000) did complete evolutionary model calculations for AmFm stars. In the absence of any tubulence, an Fe convection zone appears at $200000 \mathrm{~K}$. Assuming the mass mixed above it, the anomalies are very similar but larger than those observed. They added turbulence in the outer stellar regions in order to improve the agreement with the observed anomalies in AmFm stars. They found the anomalies to depend, besides age, on only one parameter, namely the depth of the zone effectively mixed by turbulence. They compared calculated surface abundances to observations of a number of recently observed AmFm stars. For Sirius A, 16 abundances (including 4 upper limits) are available for comparison (see Fig. 18 of Richer et al. 2000). Of these, 12 are well 
reproduced by the model, while 3 are not so well reproduced and one is a very uncertain observation.

In cluster AmFm stars, the age and initial abundances are known. There is then less arbitrariness in the calculations but fewer chemical species have been observed than in Sirius. The available observations (Hyades, Pleiades and Praesepe stars were compared) agree reasonably well with the calculated models for the five stars which were compared (see Fig. 19 to 23 of Richer et al. 2000). The zone mixed by turbulence must be slightly deeper than the iron convection zone, reducing the abundance anomalies to values which are too small for iron peak convection zones to develop in most models. The origin of the mixing process remains uncertain.

There is considerable scatter in the observations between different observers so that it is premature to conclude that hydrodynamical processes other than turbulence are needed to explain the observations. This is not ruled out but the observations do not appear good enough to establish it. Given the potential to constrain interior stellar hydrodynamics, it appears that a major effort is needed to improve abundance determinations of AmFm stars. For Sirius, for example, the current uncertainties (see Fig. 18 of Richer et al. 2000) can certainly be reduced. The whole spctrum (visible and UV) should be observed in order to use as many lines as possible for as many atomic species as possible.

The pulsational properties of these models are compatible with the observed pulsational properties of $\delta$ Scuti, $\delta$ Delphini and AmFm stars (see Turcotte et al. 2000): the AmFm stars closest to the main sequence do not pulsate while more evolved ones sometimes do.

\section{Population II}

\subsection{Turnoff Stars}

Calculations were first carried out for a metal poor cluster, M92 (Richard et al., $2002 \mathrm{~b}$ ). It was shown that, at least in a $0.8 M_{\odot}$ star, it is a better approximation not to let $\mathrm{Fe}$ diffuse than to calculate its gravitational settling without including the effects of $g_{R}(\mathrm{Fe})$.

In the absence of any turbulence outside of convection zones, the effects of atomic diffusion are large mainly for stars more massive than $0.7 M_{\odot}$. Overabundances are expected in some stars with $T_{\text {eff }} \geq 6000 \mathrm{~K}$. Most chemical species heavier than CNO are affected. At 12 Gyr (see Fig. 10 and 13 of Richard et al., $2002 \mathrm{~b}$ ), overabundance factors may reach 10 in some cases (e.g. for $\mathrm{Al}$ or $\mathrm{Ni}$ ) while others are limited to 3 (e.g. for Fe).

In Richard et al. (2002a), it is shown that while radiative accelerations and gravitational settling may lead to abundance anomalies by factors of 2 to 10 in turnoff stars of metal poor clusters such as M92, much smaller abundance anomalies are expected in relatively metal rich globular clusters such as M5, M71 or 47 Tuc. Even in NGC 6397, which is only a factor of 2 more metal rich than M92, atomic diffusion is expected to lead to smaller anomalies than in M92 (see Fig. 10 and 11 of Richard et al. 2002a). In field stars with $T_{\text {eff }} \geq 6000 \mathrm{~K}$ and $[\mathrm{Fe} / \mathrm{H}]<-2.3$, the abundance anomalies might be even larger than in M92. Reduction of metallicity beyond $[\mathrm{Fe} / \mathrm{H}]=-3.31$ is shown not to cause further 

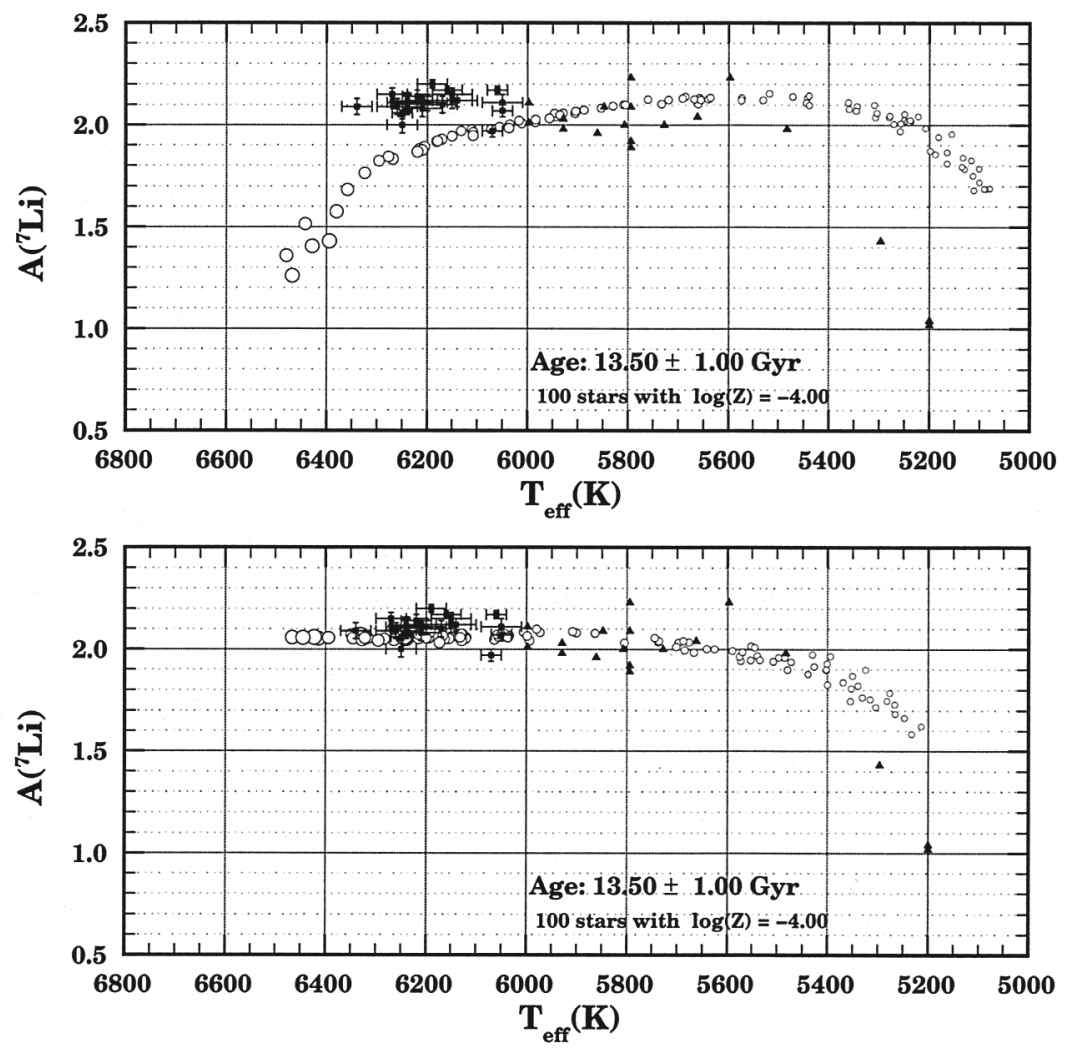

Figure 1. Predicted Li abundance in stars without turbulence (upper part) and with just enough turbulence to minimize $\mathrm{Li}$ abundance reduction. The size of circles is a function of the radius of the stars in order to indicate roughly their evolutionary stage. For the calculations, the initial value of $\mathrm{A}\left({ }^{7} \mathrm{Li}\right)$ is 2.3 . Further description is found in the text. Also shown are observations in metal poor halo stars by Spite et al. (1984; triangles) and by Ryan et al. (1999; squares with error bars).

structural changes to models (see Fig. 1 of Richard et al. 2002a). Below that metallicity, all metals may be treated as trace elements.

The calculated surface abundances were compared to recent observations of abundances in globular clusters as well as to observations of Li in halo stars. On the upper part of Figure 1 is shown the calculated surface Li concentration (open circles) in 100 stars of initial metallicity $\log Z=-4$. These are the result of a Monte Carlo simulation based on interpolations among a dozen complete evolutionary tracks (see Fig. 15 of Richard et al. 2002a for the results of a different draw in which $Z$ was also allowed to vary). The age of stars was randomly generated around 13.5 Gyr with a gaussian distribution of 1.0 Gyr standard deviation. No turbulence is assumed outside of convection zones. Observations of $\mathrm{Li}$ abundance in metal poor halo stars by Spite et al. (1984; filled triangles) and by Ryan et al. (1999; filled squares with error bars) are also shown. No effort was made to fit the Li observations below $5500 \mathrm{~K}$ since these may have been 
affected by pre-main sequence evolution. However it does not appear possible to avoid a progressive reduction of the $\mathrm{Li}$ abundance as $T_{\text {eff }}$ increases above 6000 $\mathrm{K}$ in our models. This is not observed. As in Pop I stars, additional turbulence appears to be required at least in some stars.

Series of models with different assumptions about the strength of turbulence were then calculated. One series minimizes the spread on the Li plateau and the result of a simulation for 100 stars is shown on the lower part of Figure 1. This shows that the high constancy of the Li abundance observations above $5600 \mathrm{~K}$ can be reproduced and even exceeded in a self consistent model with turbulence. Even when turbulence is adjusted to minimize the reduction of $\mathrm{Li}$ abundance, there remains a reduction by a factor of at least 1.6 from the original $\mathrm{Li}$ abundance. The surface metal abundance is reduced by at least 0.1 dex. Independent of the degree of turbulence in the outer regions, gravitational settling of $\mathrm{He}$ in the central region reduces the lifetime of Pop II stars by 4 to $7 \%$ depending on the criterion used. VandenBerg et al. (2002) show that this leads to a 1.5 Gyr reduction of the age of M92.

Comparisons were made to abundance observations of metals in a number of clusters by Richard et al. (2002b) and Richard et al. (2002a) to determine if hydrodynamical processes competing with atomic diffusion are required by observations of metals. For most metals the situation remains ambiguous: observations, taking into account the error bars, do not yet require additional processes. Monte Carlo simulations show that the Spite plateau for Li in low metallicity field stars remains the strongest argument for the presence of a process competing with atomic diffusion. Salaris \& Weiss (2001) question this conclusion. They consider it is premature until larger samples of stars are available or it is confirmed by the observations of metals in turnoff stars of clusters.

The turbulent transport coefficients required in the Sun to lead to the observed Li abundance (Proffitt \& Michaud, 1991) and in Pop II stars to minimize Li destruction are shown in Figure 2. They are surprisingly similar. This should not be viewed as implying a fundamental value to these coefficients. It merely shows similar mixing in both cases.

\subsection{Horizontal Branch}

It was noticed by Sargent \& Searle $(1967,1968)$ that field halo stars have abundance anomalies similar to those of some main sequence HgMn stars when both types of stars have the same $T_{\text {eff }}$ and $\log g$. This occurs where the horizontal branch crosses the main sequence. The $\mathrm{P}$ lines in Feige 86 served as a strong link between the abundances in field horizontal branch Pop II stars and HgMn stars. It was then argued that the small He abundance in those stars could not be used to argue that they had an He abundance smaller than the cosmological abundance. The observation of a relative overabundance of ${ }^{3} \mathrm{He}$ by Hartoog (1979) confirmed the link with the main sequence star 3 Cen A which had been strengthened by the comparison of the observations of 14 chemical species in both stars by Baschek \& Sargent (1976).

Subsequent observations supported that point of view. Glaspey et al. (1989) measured an underabundance of $\mathrm{He}$ and an overabundance of $\mathrm{Fe}$ by a factor of 50 in one $T_{\text {eff }}=16000 \mathrm{~K}$ horizontal branch star of NGC 6752 but not in cooler HB stars of the same cluster. This observation has now been strikingly con- 


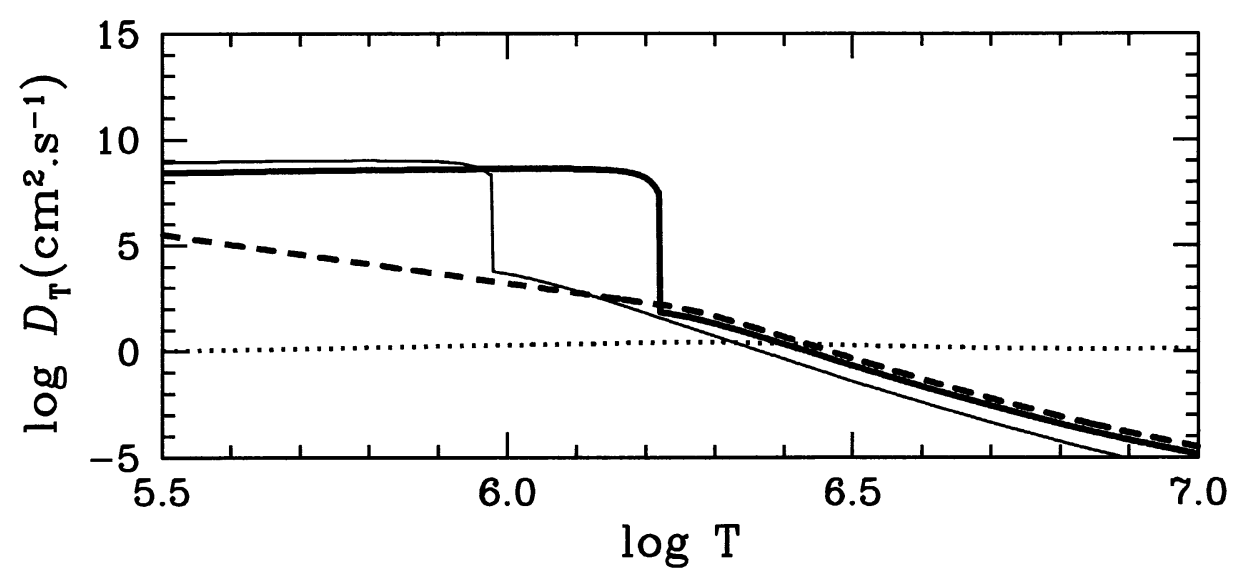

Figure 2. Turbulent transport coefficients in a $0.7 M_{\odot},[\mathrm{Fe} / \mathrm{H}]=-2.31$, Pop II star (continuous thick line) and in a $0.8 M_{\odot}$ (continous thin line) both of $4.3 \mathrm{Gyr}$ as well as in the Sun (dashed line). The dotted line is the He atomic diffusion coefficient.

firmed by Behr et al. (1999, 2000a,b) and Moehler et al. (2000) who observed many horizontal branch stars of NGC6752, M15 and M13 and obtained that whereas those cooler than about $11000 \mathrm{~K}$ have the same composition as giants, those hotter than $11000 \mathrm{~K}$ usually have larger abundances of some metals by large factors. They observed in particular Fe to be overabundant by a factor of 50 (see Fig. 1 of Behr et al. 1999). Since such anomalies cannot have been produced inside those stars and all HB stars must have had very similar original composition, this is a striking confirmation of the importance of transport processes in that region of the HR diagram. The link to transport processes is further strenghtened by the fact that the higher $T_{\text {eff }}$ HB stars rotate more slowly than the cooler ones which show no abundance anomalies (Behr et al. 2000a,b; Recio-Blanco et al. 2002). For a review see Moehler (2001).

Competition between atomic diffusion and meridional circulation. Michaud et al. (1983) considered atomic diffusion processes in horizontal branch stars. If one assumes that there is no mixing process outside of convection zones and no large mass loss rate, they showed that overabundances by factors of order 100 would be created in the atmosphere by atomic diffusion driven by differential radiative acceleration. Anomalies could be present from at least $T_{\text {eff }}=6300$ $\mathrm{K}$ to the hottest HB stars. They showed how both mass loss and mixing due to rotation should limit the anomalies. They also discussed how competing hydrodynamical processes, and in particular turbulence, could limit abundance anomalies on the HB. In their analysis of two stars of NGC 6752, Glaspey et al. (1989) used their rotation velocities to suggest a link between abundance anomalies, rotation velocities and $\log g$ (see their section IV). Michaud (1991) used the same arguments to predict that abundance anomalies should be expected in HB stars hotter than $11000 \mathrm{~K}$ (see his section 7). The arguments he used are summarized below. 


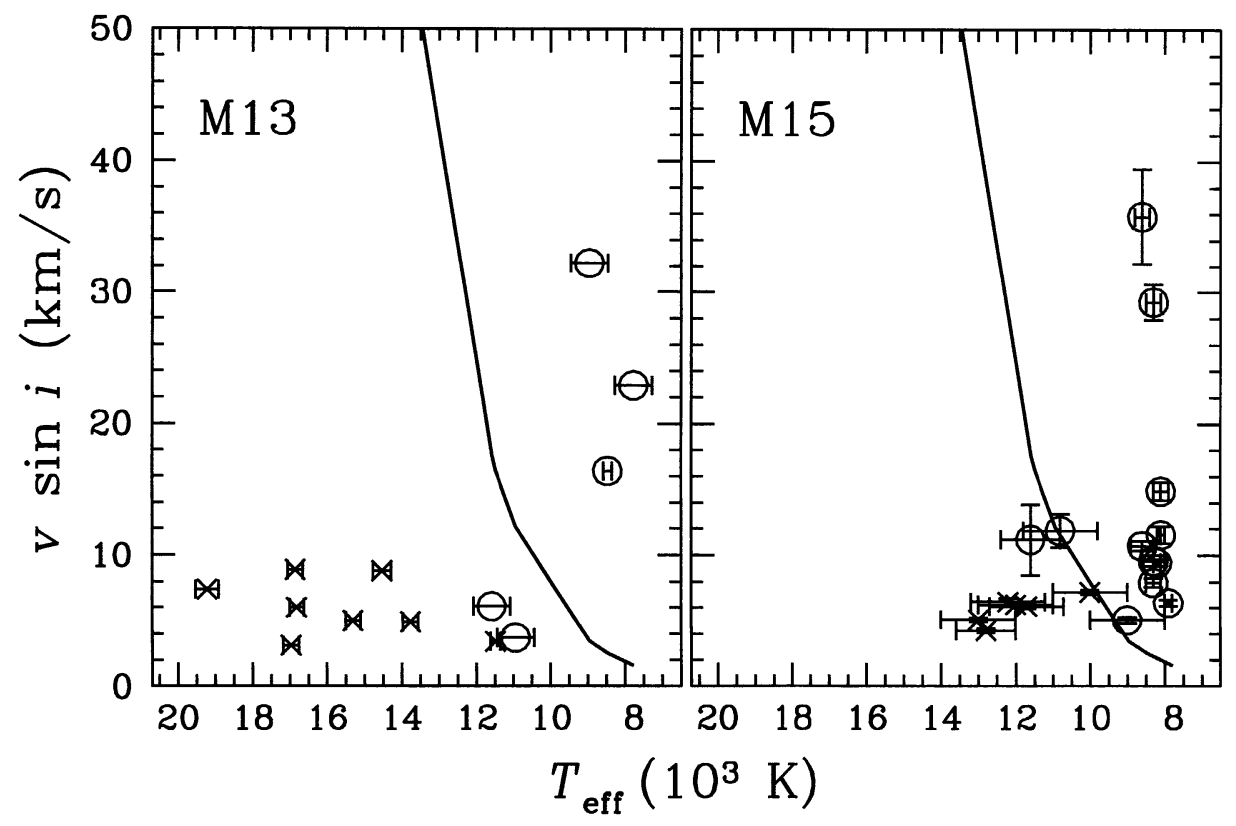

Figure 3. Rotation velocities as a function of $T_{\text {eff }}$. Open circles identify stars with the same Fe abundance as giants while crosses identify those with an overabundance of $\mathrm{Fe}$ by a factor of 50 . The continuous curve gives the limiting equatorial rotation velocity above which stars should be too well mixed for anomalies to develop. The data is from Behr et al. (1999, 2000a,b) For M13, $v \sin i$ error bars are very short and are not shown. If one could correct for $\sin i$, all open circles should be to the right of the curve and all crosses to the left.

Where the main sequence and the HB cross, the HgMn phenomenon has (Abt 2000 and references therein) an equatorial rotational velocity upper limit, $V_{e q}^{l}$, of $100 \mathrm{~km} \mathrm{~s}^{-1}$. Furthermore, Michaud (1982) obtained, using the ratio of diffusion and meridional circulation velocities, that meridional circulation strongly reduces expected abundance anomalies in $\mathrm{HgMn}$ stars if the equatorial rotation velocity is larger than $100 \mathrm{~km} \mathrm{~s}^{-1}$. The transport processes caused by rotation, may be parametrized by the Eddington-Sweet velocity (Sweet, 1950; Tassoul, 1978), which, neglecting the second cell, may be expressed for scaling purposes as:

$$
U_{E S}=\frac{V_{e q}^{2} R^{3} L}{G M_{r} M^{2}} \propto \frac{V_{e q}^{2} T_{e f f}^{4}}{M^{0.5} g^{2.5}} .
$$

On the other hand, at given $T$ and $\rho$, the diffusion velocity is $\propto g$. Assuming that the same value of the ratio of the diffusion and circulation velocities determines the limiting velocity $V_{e q}^{l}$ in HgMn and HB stars, then it should scale as

$$
V_{e q}^{l} \propto g^{1.75} / T_{e f f}^{2}
$$

along the HB (see also Fig. 1 of Michaud (1982) and Fig. 3 of Charbonneau \& Michaud 1988). 
HB stars have at $T_{\text {eff }}=15000 \mathrm{~K}$ approximately the same $\log g=4.3$ as main sequence stars. The same limiting equatorial rotation velocity may be used there. Using Eq. (2) and the observed $T_{\text {eff }}$ and $\log g$ of Behr et al. (1999) for M13, we have determined the limiting velocities shown on Fig. 3 by anchoring Eq. (2) using $100 \mathrm{~km} \mathrm{~s}^{-1}$ at $15000 \mathrm{~K}$. The same limiting velocity as a function of $T_{\text {eff }}$ is shown for M15. If one could correct for $\sin i$, open circles should be to the right of the curve and crosses to the left. The agreement seems very good. Given the observed rotation velocities, abundance anomalies in M13 and M15 are expected in stars hotter than $11000 \mathrm{~K}$ as observed.

The calculations of Michaud (1982) and of Charbonneau \& Michaud (1988) were done with the first order meridional circulation patterns of Tassoul \& Tassoul (1982). These results could be modified by using circulation patterns determined to the second order (Tassoul \& Tassoul, 1995).

The above discussion is based on scaling calculations done in Pop I main sequence stars. They should be repeated in HB models. However given the very rapid decrease of $V_{e q}^{l}$ with $T_{e f f}$ around $11000 \mathrm{~K}$ on the $\mathrm{HB}$, the exact value of the limiting velocity may not be so critical.

\section{Constraints on Stellar Turbulence and Rotational Mixing}

There appears to be an upper limit to the rotation velocity of AmFm and $\mathrm{HgMn}$ stars beyond which the phenomenon disappears (see sec. 2.). On the horizontal branch of globular clusters there is again an upper limit to the rotation velocity beyond which no anomalies are present. How rotation interferes with the development of abundance anomalies is not fully understood but the link to meridional circulation has led to a $\log g$ dependence of this limit (see sec. 3.2) and to expect that anomalies would be found in HB stars hotter than $11000 \mathrm{~K}$.

Beyond that, a link between abundance anomalies and rotation is not established in Pop I main sequence F, A and late B Pop I stars nor in HB and turnoff Pop II stars. From a comparison of observations to a large number of calculated evolutionary tracks (close to a thousand have been calculated), it is however possible to impose a few constraints on stellar hydrodynamics assuming that turbulence, whatever its cause, is the main process limiting anomalies:

1) In AmFm stars (Richer et al. 2000) spanning the range $7000<T_{\text {eff }}<$ $10000 \mathrm{~K}$ the mixed mass $\sim 10^{-5} M$ and the anomalies $\sim \times 10$.

2) Furthermore, since it takes some $10^{8} \mathrm{yr}$ to establish the anomalies, the maximum allowed mass loss rate is of order $\sim 10^{-5} M / 10^{8} \mathrm{yr} \sim 10^{-13} M_{\odot} \mathrm{yr}^{-1}$.

3) For stars to have anomalies $<0.1$ dex, it is necessary (Turcotte et al., 1998a) that the mixed mass be $\geq 10^{-3} M$.

4) For the $\mathrm{p}+\mathrm{Li}$ reaction to lead to $\mathrm{Li}$ destruction by $\times 10^{-2}$, a mixed mass $\sim 10^{-2} M$ is needed in the Sun and in Li gap stars $\left(T_{e f f} \sim 6700 \mathrm{~K}\right.$ ) (see Richard, Michaud, Richer, and Talon, in preparation).

5) In $\mathrm{F}$ or $\mathrm{A}$ stars, for $\mathrm{Li}$ not to be destroyed, the mixed mass must be $<10^{-2}$ while for metals to be normal it must be $>10^{-3} M$ so that those stars whose mixed mass remains within that range throughout their main sequence evolution would be normal stars with their original $\mathrm{Li}$. 
6) Within a factor of $\sim 10$, the same formula, $D_{T}=D_{0}\left(\rho / \rho_{0}\right)^{-n}$, leads to the observed Li destruction in the Sun and minimizes Li abundance reduction in Pop II stars (see sec. 3.1.).

7) For Be to be destroyed by less than $\times 2$ in the Sun, one needs $n \geq 3$. According to Balachandran (this volume) even a factor of 2 reduction is excluded by observations. If confirmed, her result implies an even more rapid decrease of turbulent transport below the convection zone than we used.

\section{References}

Abt, H. A. 2000, ApJ, 544, 933

Bahcall, J. N., Pinsonneault, M. H., \& Wasserburg, G. J. 1995, Rev. Mod. Phys., 67, 781

Baschek, B. \& Sargent, A. I. 1976, A\&A, 53, 47

Basu, S. 1997, MNRAS, 288, 572

Behr, B. B., Cohen, J. G., \& McCarthy, J. K. 2000a, ApJ, 531, L37

Behr, B. B., Cohen, J. G., McCarthy, J. K., \& Djorgovski, S. G. 1999, ApJ, 517, L135

Behr, B. B., Djorgovski, S. G., Cohen, J. G., McCarthy, J. K., Côté, P., Piotto, G., \& Zoccali, M. 2000b, ApJ, 528, 849

Brun, A. S., Turck-Chieze, S., \& Zahn, J. P. 1999, ApJ, 525, 1032

Burgers, J. M. 1969, Flow Equations for Composite Gases (New York: Academic Press)

Charbonneau, P. \& Michaud, G. 1988, ApJ, 334, 746

Christensen-Dalsgaard, J., Proffitt, C. R., \& Thompson, M. J. 1993, ApJ, 403, 75

Glaspey, J. W., Michaud, G., Moffat, A. F. J., \& Demers, S. 1989, ApJ, 339, 926

Guenther, D. B., Kim, Y.-C., \& Demarque, P. 1996, ApJ, 463, 382

Guzik, J. A. \& Cox, A. N. 1992, ApJ, 386, 729

Hartoog, M. R. 1979, ApJ, 231, 161

Michaud, G. 1982, ApJ, 258, 349

Michaud, G. 1991, in IAU Symposium 145, Evolution of Stars: the Photospheric Abundance Connection, Golden Sands, Bulgaria, ed. G. Michaud \& A. Tutukov (Doldrecht: Kluwer), 111-124

Michaud, G., Vauclair, G., \& Vauclair, S. 1983, ApJ, 267, 256

Moehler, S. 2001, PASP, 113, 1162

Moehler, S., Sweigart, A. V., Landsman, W. B., \& Heber, U. 2000, A\&A, 360, 120

Proffitt, C. R. \& Michaud, G. 1991, ApJ, 380, 238

Recio-Blanco, A., Piotto, G., Aparicio, A., \& Renzini, A. 2002, ApJ, 572, L71

Richard, O., Michaud, G., \& Richer, J. 2001, ApJ, 558, 377

-. 2002a, ApJ, 580, 1100

Richard, O., Michaud, G., Richer, J., Turcotte, S., Turck-Chieze, S., \& VandenBerg, D. A. $2002 \mathrm{~b}, \mathrm{ApJ}, 568,979$

Richard, O., Vauclair, S., Charbonnel, C., \& Dziembowski, W. A. 1996, A\&A, 312, 1000

Richer, J., Michaud, G., Rogers, F., Iglesias, C., Turcotte, S., \& LeBlanc, F. 1998, ApJ, 492,833

Richer, J., Michaud, G., \& Turcotte, S. 2000, ApJ, 529, 338

Ryan, S. G., Norris, J. E., \& Beers, T. C. 1999, ApJ, 523, 654

Salaris, M. \& Weiss, A. 2001, A\&A, 376, 955

Sargent, W. L. W. \& Searle, L. 1967, ApJ, 150, L33 
-. 1968, ApJ, 152, 443

Spite, M., Maillard, J.-P., \& Spite, F. 1984, A\&A, 141, 56

Sweet, P. A. 1950, MNRAS, 110, 548

Tassoul, J.-L. 1978, Theory of Rotating Stars (Princeton: Princeton University Press)

Tassoul, J.-L. \& Tassoul, M. 1982, ApJS, 49, 317

Tassoul, M. \& Tassoul, J.-L. 1995, ApJ, 440, 789

Turcotte, S., Richer, J., \& Michaud, G. 1998a, ApJ, 504, 559

Turcotte, S., Richer, J., Michaud, G., \& Christensen-Dalsgaard, J. 2000, A\&A, 360, 603

Turcotte, S., Richer, J., Michaud, G., Iglesias, C., \& Rogers, F. 1998b, ApJ, 504, 539

VandenBerg, D. A., Richard, O., Michaud, G., \& Richer, J. 2002, ApJ, 571, 487

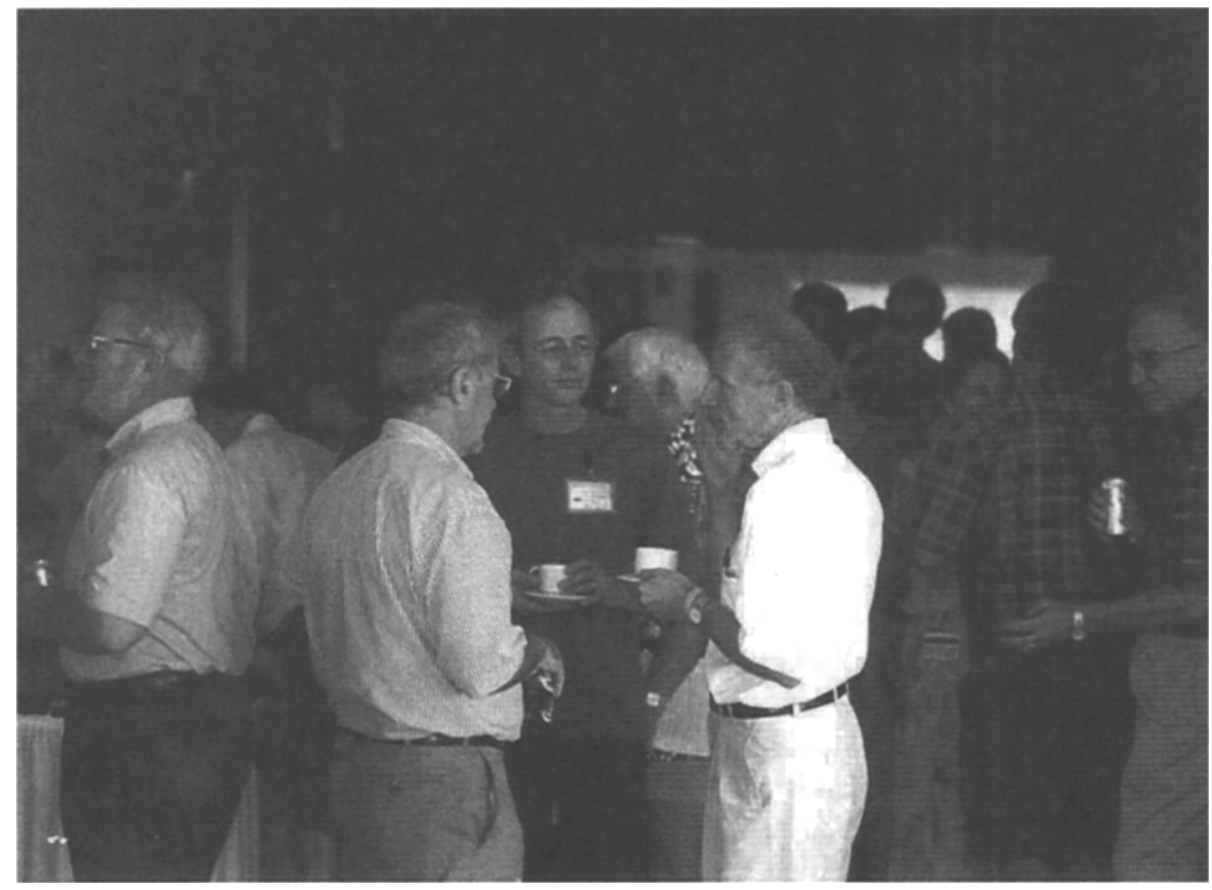

Robert Deupree, Andre Maeder, Georges Meynet, Georges Michaud and Sabatino Sofia. 